\title{
Transcription Factor Gene
}

National Cancer Institute

\section{Source}

National Cancer Institute. Transcription Factor Gene. NCI Thesaurus. Code C20420.

Genes that code for transcription factors. 